\title{
Successful treatment of a 67-year-old woman with urethral adenocarcinoma with the use of external beam radiotherapy and image guided adaptive interstitial brachytherapy
}

\author{
Jasmin Mujkanovic, MSl, Kari Tanderup, Phd', Mads Agerbaek, MD', Ulla Bisgaard, MD², Søren Høyer, MD, PhD³, \\ Jacob Christian Lindegaard, MD, DMSc', Lars Fokdal MD, PhD' \\ 'Department of Oncology, ${ }^{2}$ Department of Urology, ${ }^{3}$ Department of Pathology, Aarhus University Hospital, Århus, Denmark
}

\begin{abstract}
Primary urethral cancer (PUC) is a very rare disease. This case report illustrates a successful treatment approach of a 67-year-old woman with a urethral adenocarcinoma selected for an organ preserving treatment with external beam radiotherapy (EBRT) and interstitial brachytherapy (BT) boost, using the GEC-ESTRO target concept originally designed for locally advanced cervical cancer (LACC). Treatment included EBRT with 45 Gy in 25 fractions followed by image guided adaptive interstitial BT (IGABT) with a pulsed-dose-rate (PDR) BT boost with 30 Gy in 50 hourly pulses. The $\mathrm{D}_{90}$ for $\mathrm{CTV}_{\mathrm{HR}}$ was $79.1 \mathrm{~Gy}$ in $\mathrm{EQD}_{3}$. At 24 months follow-up, the patient was recurrence free and without
\end{abstract} treatment related side effects.

J Contemp Brachytherapy 2016; 8, 5: 434-437 DOI: 10.5114/jcb.20l6.63461

Key words: interstitial brachytherapy, primary urethral cancer, radiotherapy.

\section{Purpose}

Primary urethral cancer (PUC) is a very rare disease with an annual age-standardized ratio of 1.5 per million women. The most common histological type is transitional cell carcinoma (55\%), squamous cell carcinoma (22\%), and adenocarcinoma (16\%). Adenocarcinomas originate from Skene glands and can be divided into several subgroups. These include papillary/micropapillary adenocarcinoma, clear cell adenocarcinoma, adenocarcinoma with intestinal differentiation, and unspecified adenocarcinoma [1,2]. Primary urethral cancer presents with symptoms such as dysuria and later hematuria or regular bleeding. Due to the female anatomy, women have an increased risk for a late diagnosis $[3,4]$.

Treatment of PUC is individualized and include surgery or radiotherapy. The surgical approach depends on tumor stage and anatomic location and often results in major surgery with a pelvic exenteration [5]. Radiotherapy is an alternative to surgery and include external beam radiotherapy (EBRT), brachytherapy alone, or a combination of the two modalities [4,6,7].

This case report describes a case of PUC treated with EBRT followed by image guided adaptive interstitial BT (IGABT) using the GEC-ESTRO (Groupe Européen de Cu- riethérapie [GEC] and European Society for Radiotherapy \& Oncology [ESTRO]) adaptive target concept originally designed for locally advanced cervical cancer (LACC) [8,9].

\section{Case report}

Sixty-seven-year-old woman presents with vaginal bleeding. Previous, a supravaginal hysterectomy was performed due to a fibroma, but apart from that, she was without comorbidity. A gynecological examination in general anesthesia, showed an exophytic tumor in the area of the orificium externum urethra (Figure 1). The tumor was infiltrating into the anterior vaginal wall, but mobile in relation to the pubic bones. The tumor dimensions were $2 \mathrm{~cm}$ in height and $4.5 \mathrm{~cm}$ in width and thickness. No lymph node metastases were found in the groins.

Biopsy showed an adenocarcinoma, with an immune profile that was positive for $\mathrm{CDK} 20+, \mathrm{CDX} 2+, \mathrm{CEA}+$, suggesting a primary origin from the large bowel.

A subsequent cystoscopy and a colonoscopy were without abnormal findings. Imaging with positron emission tomography - computed tomography (PET-CT) showed intense metabolic activity in the vulvar region in relation to the urethra, but no lymph node or distant metastasis. Magnetic resonance imaging (MRI) showed a solid tumor

Address for correspondence: Lars Fokdal, MD, PhD, Department of Oncology, Århus University Hospital, Received: 12.07 .2016 DK-8000 Århus C, Denmark, phone: +45 41273606, 凶e-mail: Larsfokd@rm.dk 

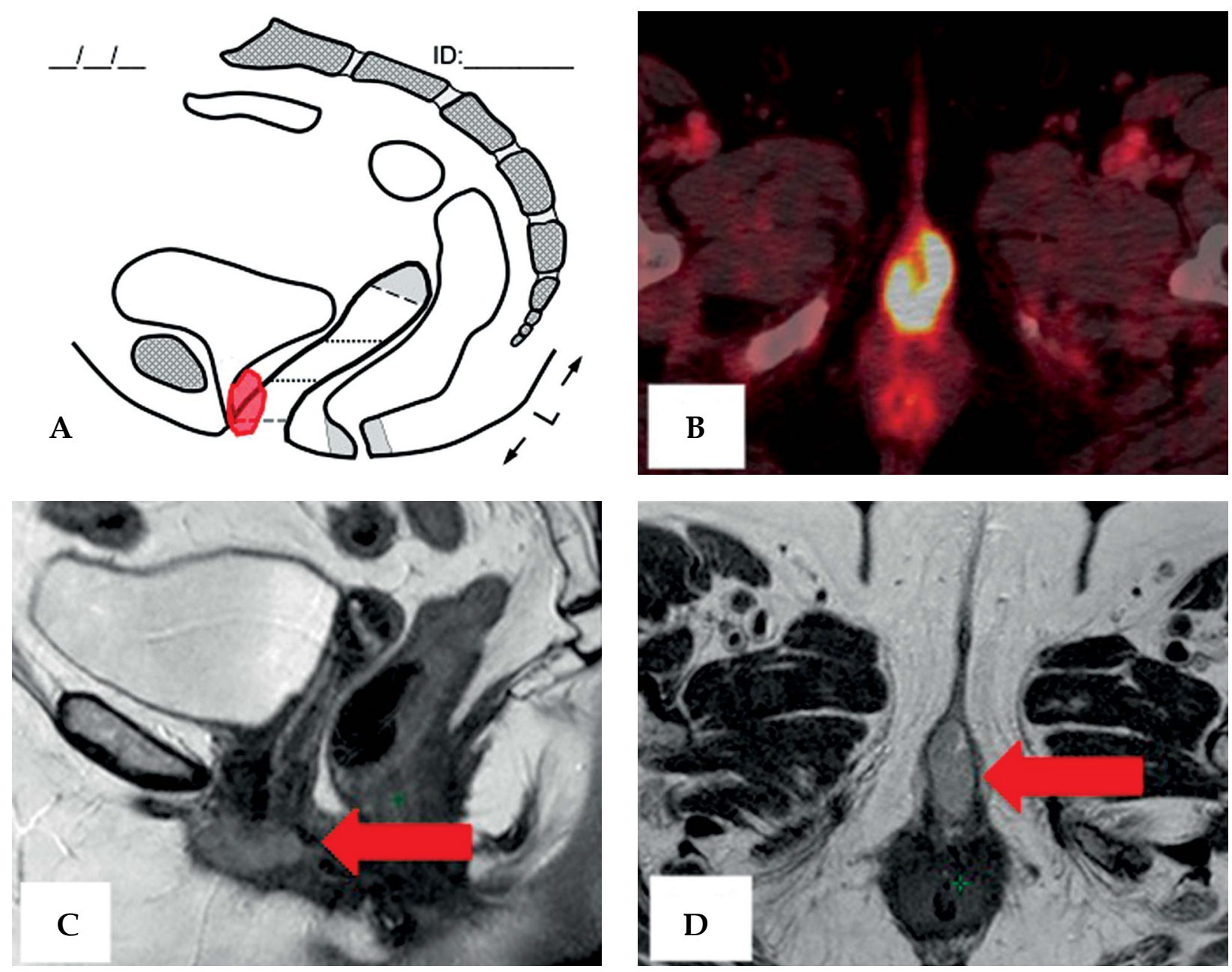

Fig. 1. Tumor presentation at time of diagnosis. A) Clinical examination showed an exophytic tumor infiltrating the anterior wall of the vagina. B) FDG PET-CT was with metabolic activity in the vulvar region in relationship to the urethra. C, D) T2 weighted MRI showed a hyper intense tumor with expansive growth pattern invading the distal part of the vagina

at the urethral meatus measuring $4.5 \times 1.4 \times 2.5 \mathrm{~cm}$ with an expansive growth pattern. The tumor was adhered to the urogenital diaphragm and involved the vagina (Figure 1).

Due to the clinical and radiological findings, it was concluded that the tumor was originating from the Skene glands and classified as a PUC. The tumor was considered operable with a complete cystectomy followed by construction of an ileal conduit. Apart from surgery, an organ-sparing treatment approach employing radiotherapy was discussed. Chemotherapy during or after radiotherapy was not considered because of lack of evidence in this rare disease. The patient was presented for the different treatment options and agreed to go for an organ sparing strategy with radiotherapy.

\section{Radiotherapy technique}

\section{External beam radiotherapy}

Treatment was initiated with pelvic EBRT to downsize the tumor for optimal BT. The elective target for EBRT (clinical target volume [CTV]-E) included pelvic and inguinal lymph nodes and CTV-T, i.e. the gross tumor volume at diagnosis (GTV-TD) with a margin of
$1 \mathrm{~cm}$ respecting natural anatomical borders. During contouring, it was ensured that the whole urethra and vagina was included in the CTV-E. An optimization volume (homogeneous-45) was contoured for later dose planning. This volume included the GTV-TD with a $1 \mathrm{~cm}$ isotropic margin respecting the CTV-E. External beam radiotherapy was delivered with volumetric modulated arc therapy (VMAT). During optimization, it was aimed to cover the target with $95-107 \%$ of prescribed dose and keep doses to organ at risk (OAR) as low as possible without compromising the target coverage. Homogeneous- 45 was used for optimization to achieve a uniform dose of $45 \mathrm{~Gy}$ in the area where BT would be delivered.

\section{Interstitial brachytherapy}

After completion of EBRT, a gynecological examination in general anesthesia was made with insertion of an MRI compatible dummy "MUPIT" (Martinez universal perineal interstitial template, Varian Medical Systems, Inc., Palo Alto, CA, USA) into the vagina. T2 weighted MRI (3 Tesla) in para-transversal, -coronal and -sagittal planes were performed (Figure 2) for preplanning of the implant 
[7]. During the preplanning procedure, the GEC-ESTRO targets $\left(\mathrm{GTV}_{\mathrm{BT}}, \mathrm{CTV}_{\mathrm{HR}}, \mathrm{CTV}_{\mathrm{IR}}\right)$ and OAR (whole urethra, bladder, rectum, and sigmoid) were defined. First, the outer wall of the OAR was contoured. Then $\mathrm{GTV}_{\mathrm{BT}}$ was contoured on the basis of areas with high signal intensity on the MRI within the tumor volume. The $\mathrm{CTV}_{\mathrm{HR}}$ included the $\mathrm{GTV}_{\mathrm{BT}}$, and surrounding grey zones in the vagina and para-vaginal space, respecting the clinical findings at the gynecological examination. The $\mathrm{CTV}_{\mathrm{IR}}$ included the initial tumor volume, and was generated based on information from MRI and clinical examination at time of diagnosis [8,9]. Finally, the pubic bones were contoured to avoid interference between needles and bone during implantation. Pre-implant treatment planning was performed on BrachyVision (Varian Medical Systems, Inc., Palo Alto, CA, USA).

After bowel preparation, the actual implantation was performed under general anesthesia. On the day of implantation, an epidural catheter was inserted for later analgesia during BT. The implantation was carried out according to the pre-implant treatment plan using the Varian MUPIT
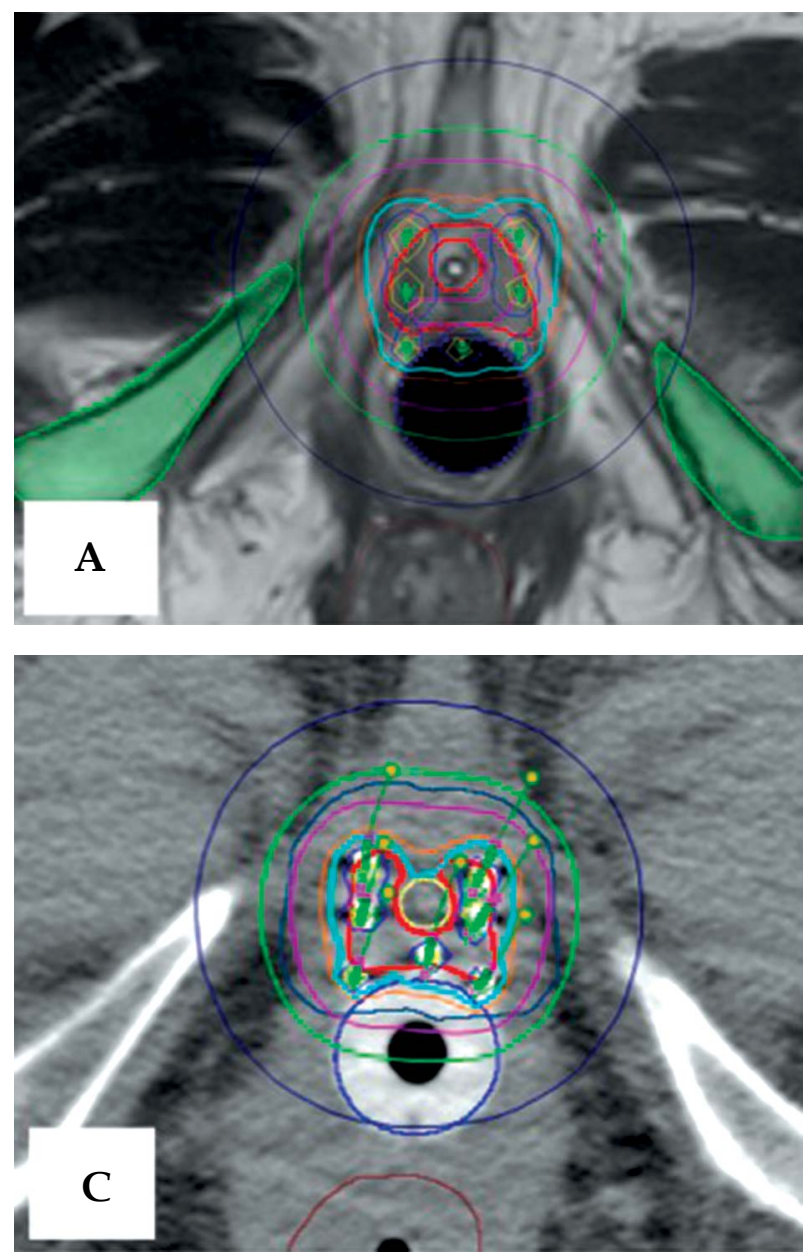

Fig. 2. Interstitial brachytherapy with MRI guided preplanning. A, B) T2 weighted MRI with a dummy MUPIT in situ was done for virtual preplanning of the implantation. The $\mathrm{GTV}_{\mathrm{BT}}, \mathrm{CTV}_{\mathrm{HR}}, \mathrm{CTV}_{\mathrm{IR}}$, and organs at risk (OAR) were contoured and preplanning of the subsequent application was conducted. C, D) A post-implant CT-scan was conducted and based on clinical information and imaging the $\mathrm{CTV}_{\mathrm{HR}}$ and $\mathrm{CTV}_{\mathrm{IR}}$ as well as organs at risk was contoured for brachytherapy planning. During manual dose optimization, it is aimed to deliver a physical dose of at least $30 \mathrm{~Gy}$ to the $\mathrm{CTV}_{\mathrm{HR}}$ and avoid double dose volumes between the needles as well as respect the constraints for each OAR template and sharp end steel needles. After implantation, a CT-scan was performed. This scan was fused with the preplanning MRI according to the MUPIT applicator. The target volumes $\left(\mathrm{CTV}_{\mathrm{HR}}\right.$ and $\left.\mathrm{CTV}_{\mathrm{IR}}\right)$ were redefined using the information from clinical examination, pre-planning MRI, and post-implant CT. Organs at risk (bladder, urethra, rectum, and sigmoid) were again outlined as they appeared on the post-implant CT (Figure 2).

\section{Planning aims and dose prescription}

The prescribed dose for EBRT was 45 Gy in 25 fractions. Planning aim of PDR-BT was to deliver a physical dose of 30 Gy in 50 hourly pulses to $\mathrm{CTV}_{\mathrm{HR}}$. The cumulative dose of EBRT and BT was calculated into equivalent dose in 2 Gy fractions $\left(\mathrm{EQD}_{2}\right)$ using $\alpha \beta=10 \mathrm{~Gy}$ for tumor, and $\alpha \beta=3$ Gy for normal tissues, and a repair half-time of $1.5 \mathrm{~h}$. Treatment planning was based on manual dose optimization with the aim of reaching a total EBRT and BT dose of $\mathrm{D}_{90}>80 \mathrm{~Gy}$ in the CTV $\mathrm{HR}$ and $\mathrm{D}_{90}>60 \mathrm{~Gy}$ in the $\mathrm{CTV}_{\mathrm{IR}}$. For OAR, the dose to $D_{2 c c}$ and the $D_{0.1 c c}$ were recorded.
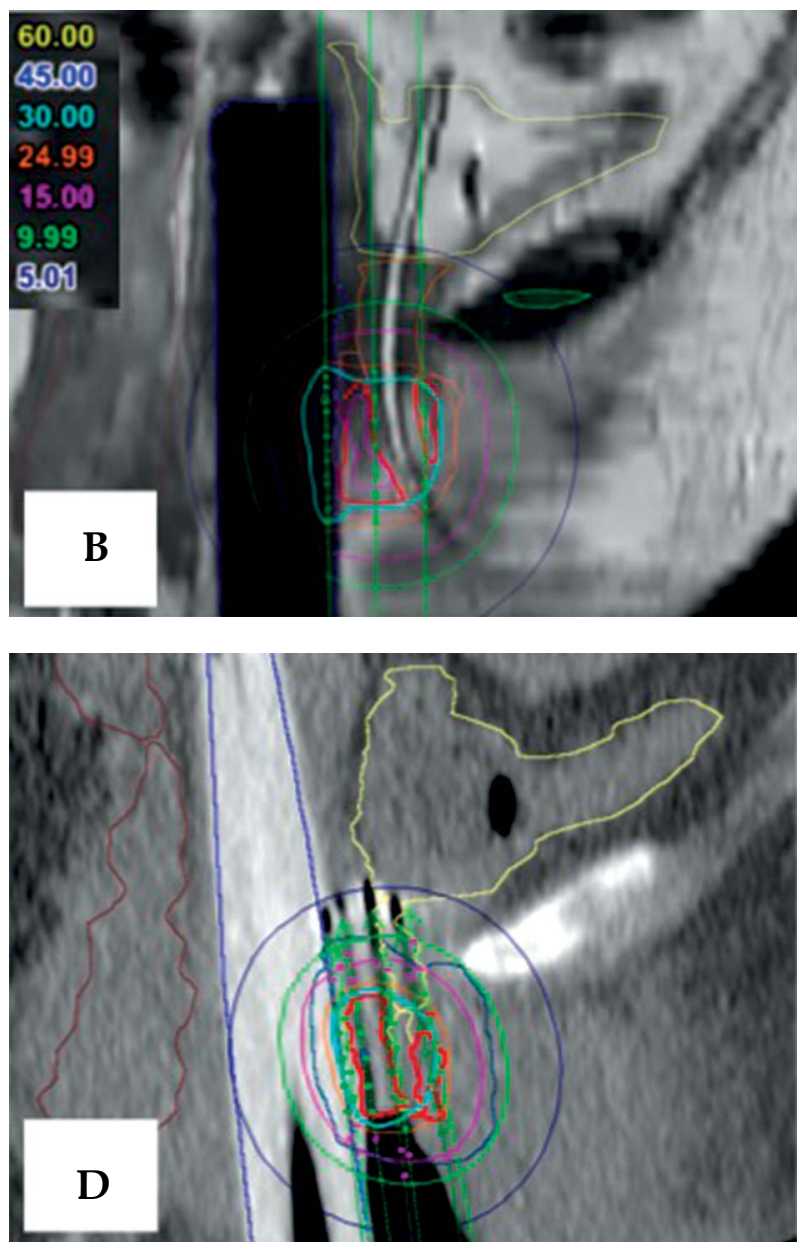
The OAR constraints used were $70 \mathrm{~Gy}$ for $\mathrm{D}_{2 \mathrm{cc}}$ rectum and sigmoid, 80 Gy for $\mathrm{D}_{2 \mathrm{cc}}$ bladder, and $98 \mathrm{~Gy}$ for $\mathrm{D}_{0.1 \mathrm{cc}}$ urethra. Basal dose was evaluated in the central plane of the $\mathrm{CTV}_{\mathrm{HR}}$ as the average dose across all basal dose points in the central plane. It was aimed to avoid double dose ( $\geq 60 \mathrm{~Gy}$ ) volumes between the needles (Figure 2).

\section{Follow-up}

Radiotherapy was well tolerated with only mild side effects including erythema in the vulvar and inguinal regions. First follow-up was conducted 3 months after the treatment with a clinical examination and imaging. During the gynecological examination, only fibrosis was found around the urethra. The PET-CT scan showed a complete metabolic response. T2 weighted MRI with diffusion weighted sequences showed only fibrosis. The latest 24 months' control showed only slight fibrosis in the periurethral tissue. The vagina was with normal dimensions. No late effects were diagnosed and the patient had not experienced any changes in urination.

\section{Discussion}

Primary urethral cancer is a rare disease without any common guideline for the treatment. In this case report, the patient was selected for an organ sparing approach with radiotherapy that included VMAT and IGABT. Development in EBRT with IMRT-technique gives an opportunity to treat a relatively large volume and at the same time, decrease the dose to OAR, thus reducing acute side effects during treatment as well as the risk for late morbidity [10].

For brachytherapy other developments have taken place. The GEC-ESTRO guidelines were designed for LACC using intracavitary or combined intracavitary/interstitial implants [8,9]. Studies have shown improvement in local control and limited morbidity after introduction of the GEC-ESTRO adaptive target concept. These findings have resulted in attempts to adapt the guidelines into the treatment of other tumors. One study reported outcome of image and laparoscopic guided interstitial brachytherapy for locally advanced primary or recurrent gynecological cancer using the adaptive GEC-ESTRO target concept. This study found an encouraging LC-rate of $92 \%$ at 3 years and only limited morbidity in 28 patients [7].

In the present case report, it was possible to adapt the GEC-ESTRO target concept and define different target volumes at time of BT, which corresponds to $\mathrm{GTV}_{\mathrm{BT}}$, $\mathrm{CTV}_{\mathrm{HR}}$, and $\mathrm{CTV}_{\mathrm{IR}}$. Furthermore, it was possible to fulfil the planning aims for the target volumes with a $D_{90}$ of $79.1 \mathrm{~Gy}$ and $64.8 \mathrm{~Gy}$ for $\mathrm{CTV}_{\mathrm{HR}}$ (volume $6.1 \mathrm{~cm}^{3}$ ) and $\mathrm{CTV}_{\mathrm{IR}}$ (volume $17.3 \mathrm{~cm}^{3}$ ), respectively. Evaluation of doses to OAR showed that we were able to respect the dose constraints that were defined with a $\mathrm{D}_{2 \mathrm{cc}}$ of $45.7 \mathrm{~Gy}$, and $44.9 \mathrm{~Gy}$, for bladder and rectum, respectively. The $\mathrm{D}_{0.1 \mathrm{cc}}$ for urethra was $85.6 \mathrm{~Gy}$. These findings indicate that the GEC-ESTRO adaptive target concept can be used in the treatment of PUC and result in good clinical results.

The literature on urethral cancer is limited, but recently a series of 10 women treated with high dose rate interstitial brachytherapy for urethral cancer (5 primary and 5 recurrent) was published. In most patients, the treatment strategy employed EBRT and image guided (CT) interstitial BT. All 5 patients with primary tumors were squamous cell histology and treatment results were acceptable with one local and one nodal recurrence, and acceptable morbidity during follow-up [11].

The case report highlights the use of EBRT and IGABT in the treatment of urethral cancer and illustrate that modern radiotherapy is a feasible option with a curative potential and limited morbidity, and should be considered in patients with PUC. However, more experience is warranted in more patients with longer follow-up to confirm the satisfying results.

\section{Conclusions}

The present case report shows that EBRT with an image guided adaptive interstitial brachytherapy boost is an attractive treatment of PUC with a possibility to provide local control, and at the same time, organ preservation with limited morbidity.

\section{Disclosure}

Authors report no conflict of interest.

\section{References}

1. Visser $\mathrm{O}$, Adolfsson J, Rossi S et al. Incidence and survival of rare urogenital cancers in Europe. Eur J Cancer 2012; 48: 456464.

2. Swartz MA, Porter MP, Lin DW et al. Incidence of primary urethral carcinoma in the United States. Urology 2006; 68: 1164-1168.

3. Carroll PR, Dixon CM. Surgical anatomy of the male and female urethra. Urol Clin North Am 1992; 19: 339-346.

4. Micaily B, Dzeda MF, Miyamoto CT et al. Brachytherapy for cancer of the female urethra. Sem Surg Oncol 1997; 13: 208-214.

5. Maggioni A, Roviglione G, Landoni F et al. Pelvic exenteration: ten-year experience at the European Institute of Oncology in Milan. Gynecol Oncol 2009; 114: 64-68.

6. Koontz BF, Lee WR. Carcinoma of the urethra: radiation oncology. Urol Clinics North Am 2010; 37: 459-466.

7. Fokdal L, Tanderup K, Nielsen SK et al. Image and laparoscopic guided interstitial brachytherapy for locally advanced primary or recurrent gynaecological cancer using the adaptive GEC ESTRO target concept. Radiother Oncol 2011; 100: 473-479.

8. Haie-Meder C, Pötter R, Van Limbergen E et al. Recommendations from Gynaecological (GYN) GEC-ESTRO Working Group (I): concepts and terms in 3D image based 3D treatment planning in cervix cancer brachytherapy with emphasis on MRI assessment of GTV and CTV. Radiother Oncol 2005; 74: 235-245.

9. Pötter R, Haie-Meder C, Van Limbergen EV et al. Recommendations from gynaecological (GYN) GEC ESTRO working group (II): Concepts and terms in 3D image-based treatment planning in cervix cancer brachytherapy-3D dose volume parameters and aspects of 3D image-based anatomy, radiation physics, radiobiology. Radiother Oncol 2006; 78: 67-77.

10. Jensen LG, Hasselle MD, Rose BS et al. Outcomes for patients with cervical cancer treated with extended-field intensity-modulated radiation therapy and concurrent cisplatin. Int J Gynecol Cancer 2013; 23: 119-125.

11. Sharma DN, Gandhi AK, Bhatla N et al. High-dose-rate interstitial brachytherapy for female peri-urethral cancer. J Contemp Brachytherapy 2016; 8: 41-47. 